\title{
High Expression of Orphan Nuclear Receptor NR4A1 in a Subset of Ovarian
}

\section{Tumors with Worse Outcome}

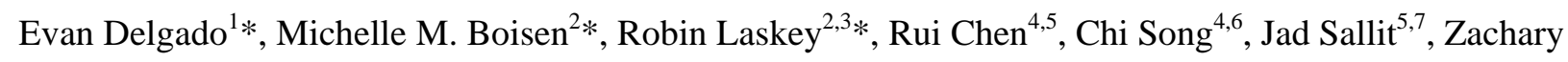
A. Yochum ${ }^{8}$, Courtney L. Andersen ${ }^{9,10}$, Matthew J. Sikora ${ }^{9}$, Jacob Wagner ${ }^{1}$, Stephen Safe $^{11}$, Esther Elishaev $^{12}$, Adrian Lee ${ }^{5}$, Robert P. Edwards ${ }^{2}$, Paul Haluska ${ }^{13,14}$, George Tseng ${ }^{4 \wedge}$, Mark Schurdak ${ }^{1 \wedge}$, Steffi Oesterreich $^{5 \wedge}$

${ }^{1}$ University of Pittsburgh Drug Discovery Institute, Pittsburgh, PA, USA; ${ }^{2}$ Division of Gynecologic Oncology, Magee-Womens Hospital of the University of Pittsburgh Medical Center, Pittsburgh, PA, USA; ${ }^{3}$ Present address: Division of Gynecologic Oncology, Summa Akron City Hospital, Akron, OH, USA; ${ }^{4}$ Department of Biostatistics and Department of Human Genetics, University of Pittsburgh, Pittsburgh, PA, USA $;{ }^{5}$ Present address: Merck \& Co, Rathway, NJ, USA; ${ }^{6}$ Present address: Division of Biostatistics, College of Public Health, The Ohio State University, Columbus, OH, USA; ${ }^{7}$ Ross University School of Medicine; ${ }^{8}$ Department of Medicine, Division of Hematology Oncology, University of Pittsburgh Cancer Institute, Pittsburgh, PA, USA; ${ }^{9}$ Department of Pharmacology and Chemical Biology, Womens Cancer Research Center, Magee-Womens Research Institute, and University of Pittsburgh Cancer Institute, Pittsburgh, PA, USA; ${ }^{10}$ Molecular Pharmacology Training Program, University of Pittsburgh School of Medicine, Pittsburgh, PA; ${ }^{11}$ Department of Veterinary Physiology and Pharmacology, Texas A\&M University, College Station, TX, USA; ${ }^{12}$ Department of Pathology, MageeWomens Hospital of the University of Pittsburgh Medical Center, Pittsburgh, PA, USA; ${ }^{13}$ Department of Oncology and Pharmacology, Mayo Clinic, Rochester, MN, USA, ${ }^{14}$ Current address: Merck Oncology, Rahway, NJ, USA

* ED, MB, and RL share first authorship, and GT, MS, and SO share senior authorship.

\author{
Corresponding author: \\ Michelle Boisen, MD \\ Division of Gynecologic Oncology \\ Magee-Womens Hospital of the University of Pittsburgh Medical Center \\ 300 Halket St. \\ Pittsburgh, PA 15217 \\ boockmeiermm@mail.magee.edu \\ Phone: 412-641-1153 \\ Fax: 412-641-5417
}


ABSTRACT

39 Objective: Nuclear receptors (NRs) play a vital role in the development and progression of several

40 cancers including breast and prostate. Using TCGA data, we sought to identify critical nuclear receptors

41 in high grade serous ovarian cancers (HGSOC) and to confirm these findings using in vitro approaches.

42 Methods: In silico analysis of TCGA data was performed to identify relevant NRs in HGSOC. Ovarian

43 cancer cell lines were screened for NR expression and functional studies were performed to determine the

44 significance of these NRs in ovarian cancers. NR expression was analyzed in ovarian cancer tissue

45 samples using immunohistochemistry to identify correlations with histology and stage of disease.

46 Results: The NR4A family of NRs was identified as a potential driver of ovarian cancer pathogenesis.

47 Overexpression of NR4A1 in particular correlated with worse progression free survival. Endogenous

48 expression of NR4A1 in normal ovarian samples was relatively high compared to that of other tissue

49 types, suggesting a unique role for this orphan receptor in the ovary. Expression of NR4A1 in HGSOC

50 cell lines as well as in patient samples was variable. NR4A1 primarily localized to the nucleus in normal

51 ovarian tissue while co-localization within the cytoplasm and nucleus was noted in ovarian cancer cell

52 lines and patient tissues.

53 Conclusions: NR4A1 is highly expressed in a subset of HGSOC samples from patients that have a worse

54 progression free survival. Studies to target NR4A1 for therapeutic intervention should include HGSOC. 
Epithelial ovarian cancer (EOC) is the most lethal gynecologic malignancy and the fifth most

57 common cause of cancer related deaths in women in the United States [1]. Despite advances in surgical

58 and chemotherapeutic strategies, there has been only a modest improvement in 5-year overall survival

59 over the past 20 years [2]. Although the majority of patients experience a response to primary therapy,

60 approximately $75 \%$ will relapse within 2 years and will ultimately undergo repetitive treatments for

61 disease recurrences until their disease becomes treatment refractory [3]. Thus, there is an unmet clinical

62 need for the development of new therapeutic strategies that will provide meaningful improvements in

63 outcomes for these patients.

EOC is a biologically heterogeneous disease and the identification of tumor-specific molecular

65 alterations may provide opportunities for targeted therapy. Biologic agents such as bevacizumab have

66 shown moderate benefit in the treatment of EOC; however, these agents can be associated with significant

67 dose-limiting toxicities, thus prompting efforts to identify less toxic therapeutic strategies [4]. In this

68 regard, nuclear receptor modulators are an attractive option due to their limited toxicity profile and ease

69 of administration, especially in patients who are unable to tolerate cytotoxic chemotherapy or other

70 biologic agents due to side effects or medical comorbidities [5, 6].

71

There are 48 known nuclear receptors (NR) that function as ligand-activated intracellular

72 transcription factors, regulating important target genes and signaling pathways. Nuclear receptors play a

73 role in many diseases including obesity, diabetes, dyslipidemia and cardiovascular disease [7, 8]. Steroid

74 hormones, thyroid hormones and vitamin D3 are some of the most well-known targets of transcriptional

75 regulation by NRs. Disruption of cell and tissue specific NR function can lead to the development and

76 progression of several cancers including breast and prostate $[9,10]$. Conversely, modulation of the

77 estrogen and androgen receptors has resulted in the successful treatment of breast cancer and prostate

78 cancer, respectively $[10,11]$. Despite what is known about NR in other malignancies, their expression

79 and function in ovarian cancer remains largely understudied. 
Using The Cancer Genome Atlas (TCGA), we sought to identify critical NRs in high-grade

81 serous ovarian cancers. We hypothesized that a subset of ovarian tumors would have similar NR

82 expression profiles suggesting a common molecular pattern of tumorigenesis. Identification of patterns of

83 NR expression may provide a potential avenue for further preclinical and clinical work which would

84 harness the therapeutic potential of targeting these receptors.

86 Materials and methods

87 Analysis of Nuclear Receptor Expression in TCGA Samples Correlation with Outcomes

Publically available TCGA data were downloaded from TCGA data portal on 3/29/2011. We combined the downloaded expression profiles from three microarray platforms, including Affymetrix Human Genome U133a, Human Exon 1.0 and Agilent G4502A, using factor analysis. We also filtered out genes with mean expression level smaller than 50 based on the Human Exon platform. A total of 504 samples were included. Then we clustered the expression profiles using a "tight clustering" algorithm developed within our institution [12]. Only NR genes were included for the clustering. The tight clustering algorithm groups similar tumors based upon expression profiles and performs re-sampling evaluation to repeatedly cluster random subsamples of data, evaluate the stability of the identified clusters, and finally generates clusters of tumors with similar gene expression patterns. Tumor samples

97 that do not exhibit gene expression patterns similar to any of the identified clusters are excluded. "Driver 98 genes" of each cluster were identified using the gene-dominant and gene-dormant index method [13].

99 This method extends the signal to noise ratio to identify features as either dominant or dormant in a 100 specific cluster compared to the other clusters. Clinical data from the TCGA were analyzed to determine clinical outcomes based on cluster.

102 Progression free survival was calculated from the date of diagnosis to the date of recurrence or censoring.

103 Overall survival was calculated from the date of diagnosis to the date of death or date of last contact.

104 Progression-free and overall survival were calculated for the patients within each cluster using Kaplan- 

124 indicated.

\section{Cell culture}

Meier methods. The Cox proportional hazard model was used to evaluate the expression of NR genes as continuous predictor variables. A p-value of $<0.05$ was considered statistically significant.

Normal human tissue mRNA, human ovarian tissue and ovarian cancer tumor specimens

The FirstChoice ${ }^{\circledR}$ Human Total RNA Survey Panel of pooled mRNA from 3 donors of 20 normal human tissues was purchased from Ambion (Austin, TX). Normal ovarian tissue and serous ovarian tumor specimens were obtained using a protocol approved by the Magee-Womens Hospital of UPMC Institutional Review Board.

The OVCAR3 and OVCAR8 cell lines were maintained in RPMI 1640 media supplemented with 10\% fetal bovine serum (FBS). OVCA432 and PEO1 cell lines were maintained in RPMI 1640 medium $+10 \%$ FBS, supplemented with L-glutamine and $2 \mathrm{mM}$ sodium pyruvate, respectively. These lines have been continuously cultured in the Division of Gynecological Oncology at MWRI. The OVSAHO cell line was obtained through the Japanese Collection of Research Bioresources and was maintained in DMEM with $10 \%$ FBS. The CA-OV-3 cell line was obtained through American Tissue Culture Collection (ATCC) and was maintained in DMEM with 10\% FBS. All cells were maintained in antibiotic free media at $37^{\circ} \mathrm{C}$ in a $5 \% \mathrm{CO} 2$ atmosphere and routinely screened for Mycoplasma contamination. All tissue culture medium and additives were purchased from Invitrogen, (Carlsbad, CA) unless otherwise

\section{Patient-derived HGSOC xenografts (PDXs)}

Patient-derived HGSOC xenografts were engrafted and propogated as previously described [14]. Briefly, tumor tissue from OVCA patients was finely minced, suspended in McCoy's 5A media (Invitrogen), engrafted into SCID/Beige mice (C.B.-17]IcrHsd-Prkdc $c^{\text {scid }} L y s t^{b g}$; Harlan) via intraperitoneal injection. Upon sacrifice, the tumors were used to either passage for subsequent studies or were collected 
131 for gene expression and immunohistochemical analysis. The PDX studies were approved by the local 132 Mayo IACUC committee.

Immunoblotting

Cells from each cell line were lysed with RIPA buffer (1\% IgePAL, 0.5\% Sodium Deoxycholate (bwt), and $0.1 \%$ SDS) on ice for 30 minutes prior to harvest for Immunoblot analysis. Protein

137 concentrations were determined via Bradford Assay (BioRad Hercules, CA) and measured using a 138 SpectroMax M5e spectrophotometer (Molecular Devices Sunnyvale, CA). Samples were run on 4-15\% 139 gradient SDS acrylamide precast gels (BioRad Hercules, CA) and transferred to PVDF membrane (EMD 140 Millipore Billerica, MA) overnight at $4^{\circ} \mathrm{C}$. Rabbit anti-NR4A1 (IMG-528 Novus Biologicals Littleton, 141 CO) and mouse anti- $\alpha$-tubulin (T9026 Sigma-Aldrich St. Louis, MO) were diluted 1:200 and 1:1000 142 respectively in 5\% non-fat dry milk/Blotto $(150 \mathrm{mM} \mathrm{NaCl}, 20 \mathrm{mM} \mathrm{pH} 7.5$ Tris-Base, and $0.1 \%$ Tween143 20) and incubated on immunoblot membranes for at least 1 hour at room temperature. Membranes were 144 subsequently washed of primary antibodies followed by probing with respective secondary antibodies at $1451: 10,000$ or 1:25,000. Clarity reagents (BioRad Hercules, CA) were used to activate HRP signals for 2 146 minutes prior to imaging using Fujifilm LAS-3000 (Fujifilm Medical Systems Stanford, CT) for 147 development.

$R N A$ extraction and $q-R T-P C R$

RNA was isolated from samples using the Illustra RNAspin Mini RNA Isolation Kit (GE

151 Healthcare, Pittsburgh, PA). iScript RT Supermix (BioRad Hercules, CA) was used to convert mRNA to 152 cDNA. The q-RT-PCR utilized SYBR green (BioRad Hercules, CA) according to manufacturer's 153 instructions. Primer sequences were: NR4A1 forward (CACATTGTTGCCAAGACCTG), NR4A1 154 reverse (TGCTGGTGTCCCATATTGG), $\beta$ actin forward (CCCTGGCACCCAGCAC), and $\beta$-actin 155 reverse (GCCGATCCACACGGAGTAC). All primers were ordered from Integrated DNA Technologies 
(Coralville, IA) with standard desalting. Each primer pair was verified using serially diluted template

157 cDNA. The fold change for each gene was calculated using the $\Delta \Delta \mathrm{Ct}$ method [15]. q-RT-PCR was

158 performed in technical triplicates for each gene of interest. A reference gene ( $\beta$-actin) was included to

159 normalize for input cDNA.

160

\section{Immunohistochemistry (IHC)}

Formalin-fixed paraffin embedded (FFPE) tissues were cut into sections of 3-5 microns. Slides were then de-paraffinized in xylene and rehydrated. Fresh antigen retrieval buffer (0.01M Sodium citrate with $0.05 \%$ Tween-20, $\mathrm{pH} 6.0$ ) was heated to boiling and the tissue slides were added to the pre-warmed buffer for 30 minutes. The tissue slides were then allowed to equilibrate to room temperature for $30 \mathrm{~min}$. Endogenous peroxidase activity was blocked by immersing slides in 3\% hydrogen peroxide solution for 5 min. After rinsing with ultrapure water, the primary antibody was added directly to the tissue sample (NR4A1, IMG-528 Novus Biologicals Littleton, CO) at 1:200 dilution in 1\% bovine serum albumin (BSA) and incubated in a humidified chamber at $4{ }^{\circ} \mathrm{C}$ overnight. The antibody was then linked using Envision Labelled Polymer-HRP Anti-Rabbit (Dako, Via Real Carpinteria, CA) for $30 \mathrm{~min}$ at RT in a humidified chamber. After rinsing, tissue was covered with Dako Cytomation DAB+ solution and incubated for 15 min before chromogen signal enhancement with DAB Sparkle Enhancer (Biocare, Concord, CA). Slides were counterstained in Harris Hematoxylin and Eosin (Sigma Aldrich) for $30 \mathrm{sec}$ and dehydrated and cleared in the routine manner. The sections were mounted in Cytoseal (VWR, Radnor, PA) with appropriate coverslips. A Nikon 90-I microscope and NIS Elements Microscope Imaging software (Nikon, Melville, NY) was then used to image the slides.

\section{Immunofluorescent Detection of Endogenous NR4A1}

Cells were plated at the following concentrations using the Multidrop Combi into polystyrene 384 well plates (Greiner, Monroe, NC): 5 x 10 4 PEO1; 2 x 10 $0^{4}$ OVCAR8 and OVCA432, 4 x $10^{4}$ OVSAHO and OVCAR3, and $2 \times 10^{4} \mathrm{CA}-\mathrm{OV}-3$. Cells were allowed to settle and attach for 30 minutes prior to 
182

moving to an incubator at $37^{\circ} \mathrm{C} / 5 \% \mathrm{CO}_{2}$. Roughly 48 hours after seeding, cells were fixed with $50 \mu \mathrm{L} /$ well fixative solution (PBS $+7.4 \%$ Formaldehyde for addition [made from 37\% Formaldehyde stock with $10 \%-15 \% \mathrm{MeOH}$ for stabilization) containing $2 \mu \mathrm{g} / \mathrm{mL}$ Hoechst added with the Mutlidrop Combi and were incubated at room temperature for 20 minutes. Wells were then washed 3 times using the Biotek plate washer with $80 \mu \mathrm{L} /$ well 1x PBS, allowing 5 minutes between washes. Following the final PBS wash, $80 \mu \mathrm{L} /$ well permeabilization buffer (PBS $+0.5 \%$ Triton $\mathrm{X}-100)$ was added and the plates were incubated at room temperature for 10 minutes. Wells were then washed with 1x PBS and $80 \mu \mathrm{L} /$ well blocking buffer (PBS $+0.5 \%$ Tween-20) was added and the plate was incubated at $4{ }^{\circ} \mathrm{C}$ overnight. After blocking, cells were incubated for 1 hour at room temperature with $25 \mu \mathrm{L} /$ well 1:100 rabbit polyclonal NR4A1 primary antibody (IMG-528 Novus Biologicals Littleton, CO) prepared in blocking buffer. Wells were washed 3 times with PBS using the Biotek plate washer. $25 \mu \mathrm{L} /$ well 1:750 goat anti-rabbit IgG secondary antibody $(\mathrm{H}+\mathrm{L})$ Dylight 550 (Thermo Fisher Scientific Pittsburgh, PA) in blocking buffer were added to each well and the plated was incubated at room temperature for 45 minutes. Wells were washed 4 times with PBS using the Biotek plate washer. 1x PBS was added to each well after the final wash and the plates were sealed. The plates were then imaged using the ImageXpress Ultra (IXU) confocal high-content imaging system (Molecular Devices Corp.).

\section{Results}

Nuclear receptor expression in TCGA serous ovarian tumor samples

Using publically available data from TCGA, we applied the previously described tight clustering algorithm to group HGSOC with similar NR expression profiles, which produced 5 distinct gene clusters (Figure 1A). Nuclear receptors within each cluster were identified as either "dominant" or "dormant" based upon gene expression levels (Table 1). We hypothesized that the overexpressed genes or "dominant drivers" within each of these clusters could potentially play critical roles in the respective subsets of HGSOC. Within the largest cluster of HGSOC (Cluster 1) the most significant driver genes contained 3 members of the NR4A subfamily of nuclear receptors: NR4A1 (Nur77, TR3), NR4A2 
(Nurr1), and NR4A3 (Nor1). Because the NR4A subfamily encompasses the dominant drivers of the

208 largest cluster of patient samples, we chose to further investigate the role and implication of these genes

209 in additional studies.

Clinical outcome of tumors with high expression of NR4A subfamily

Clinical outcomes of patients included in each cluster were compared to determine whether NR 213 expression differences are associated with patient outcomes. Patients with tumors in Cluster 1 who 214 highly expressed NR4A family members had significantly $(\mathrm{p}=0.03)$ shorter progression free survival 215 (PFS) compared to the remaining 4 clusters (Fig 1B). Median overall survival for patients in Cluster 1 216 exhibited a trend toward worse outcomes as compared to Clusters 2-5 but did not reach statistical 217 significance ( $\mathrm{p}=0.09$, Fig 1B). Subsequently, a Cox proportional hazard model was used to determine the 218 individual contribution of each of the 3 NR4A to PFS. Of the three genes, only NR4A1 was found to be 219 significantly associated with worse PFS ( $\mathrm{p}=0.0357)$. Given these findings, we sought to further investigate the role of NR4A1 in pathogenesis of HGSOC.

We first queried NR4A1 expression in the normal ovary relative to other tissues. Therefore,

224 human pooled mRNA was obtained from 21 non-diseased human tissues and qRT-PCR was performed.

225 As compared to the majority of tissue types investigated, the expression of NR4A1 in the ovary is

226 relatively high, with only skeletal muscle and tracheal tissue having higher levels of expression (Figure

227 2A). Figure 2B demonstrates IHC staining of normal ovarian tissue for NR4A1 and demonstrates nuclear 228 localization of NR4A1. In addition, given that many ovarian cancers are thought to originate in the 229 fallopian tube, we also investigated NR4A1 expression in the normal fallopian tube. As shown, similar to 230 ovarian tissue, NR4A1 staining is primarily nuclear, and is limited to the tubal epithelium. In addition, 231 our in silico analysis of Human Protein Atlas RNA seq data confirmed high expression of NR4A1 mRNA 232 in the fallopian tube (data not shown). 
To study the role of NR4A1 in ovarian cancer, we sought to compare the IHC staining pattern of normal ovarian tissue to that of patients with ovarian cancers (Figure 3). In addition to HGSOC, we also included FFPE samples with normal ovaries, and mucinous and endometrioid histologies. Of the 10

236 tissue samples from patients with normal ovaries, all stained positive for NR4A1. In each of those cases, 237 NR4A1 was localized to the nucleus with no cytoplasmic staining noted. In contrast, the majority of the 238 mucinous and HGS HGSOC showed significant cytoplasmic staining, in addition to the nuclear staining.

239 Of the 4 endometrioid tumors included in this study, one exhibited nuclear positivity for NR4A1, and 2 of these tumors were noted to have cytoplasmic positivity.

Given that NR4A1 localization seems to differ between normal ovarian tissue and ovarian cancer specimens on IHC staining, we identified several serous ovarian cancer cells lines in which to further investigate these differences using preclinical models. Figure 4 shows an immunoblot for NR4A1 in 6 different HGSOC cancer cell line models. 4 The OVCA432 and OVCAR3 cell lines have significantly higher protein levels of NR4A1 than the CA-OV-3, OVCAR8, OVSAHO, and PEO1 cell lines. Of note are the two isoforms of NR4A1 on the immunoblot. There are multiple transcript variants of this protein

247 that have been described and the role for each variant has not been clearly elucidated [16]. In order to 248 further characterize heterogeneous NR4A1 nuclear or cytoplasmic localization, we used

249 immunofluorescence analysis to evaluate the level and localization of NR4A1 in individual cells in these 250 HGSOC cell lines (Figure 4C). We detected significant heterogeneity in the degree to which there is 251 cytoplasmic positivity for NR4A1. Figure 4D demonstrates that OVCA432, OVCAR8, and CA-OV-3 252 cells have the highest levels of cytoplasmic NR4A1 staining with the median number of cells showing 253 over 50\% NR4A1 in the cytoplasm. Heterogeneous localization of NR4A1 is important when 254 considering which cell population to focus on while studying the mechanisms related to the NR4A1 255 nuclear to cytoplasmic translocation and its potential implications on resulting phenotypes. 
Data from TCGA indicates NR4A1 is upregulated in a specific subset of HGS ovarian cancer patients. In an attempt to externally validate this, we analyzed the mRNA expression of NR4A1 in a

262 series of ovarian cancer subtype PDX models (Figure 5A). As with TCGA cases and ovarian cancer cell

263 line models, PDXs displayed wide range of NR4A1 expression. NR4A1 expression did not correlate with

264 histologic subtype of the PDX or with stage of the original tumor; however, sample size was limited.

\section{Discussion} research of the role of NRs in ovarian tumorigenesis and ultimately allow for development of a targeted therapies. We identified the NR4A family of receptors, NR4A1 in particular, as portending a worse prognosis for patients within the TCGA dataset. The NR4A family members NR4A1, NR4A2, and NR4A3, are a group of closely related nuclear receptors with no known endogenous ligand ("orphan receptors"). This subgroup has been implicated in cell cycle regulation, neurological disease,

272 inflammation and carcinogenesis [17]. The overexpression of genes within the NR4A subgroup has been 273 documented in several other cancers including colon, pancreatic and lung [18-20]. Further, in bladder

274 cancer, high NR4A2 expression is associated with high tumor grade, risk for distant metastasis, and 275 overall patient survival [21].

277 for its role in carcinogenesis and apoptosis. NR4A1 overexpression in breast cancer cell line xenografts

278 results in altered inflammatory response and increased risk for metastatic disease [29]. However, 279 cytoplasmic NR4A1 can contribute to apoptosis of breast cancer cells [22]. We were able to show that 280 NR4A1 expression at baseline is relatively high within normal ovarian tissue as compared to a number of 281 other human tissue types. Comparing IHC staining profiles between normal ovarian tissue and epithelial 282 ovarian cancers, staining intensity appears stronger for the cancer specimens where NR4A1 localizes to 283 both the nucleus and cytoplasm as compared to normal ovarian tissue where cellular localization is 
nuclear only. Similarly, immunoblot analysis and immunofluorescence analysis of several HGSOC cell lines reveals significant heterogeneity both in NR4A1 protein levels as well as subcellular localization. Identifying a subpopulation of ovarian cancer patients with elevated levels of NR4A1 is critical to the future development of novel targeted therapies against NR4A1. A recent elegant study by Wilson and colleagues showed that NR4A1 contributes to platinum response in ovarian cancer and this is associated with platinum-induced nuclear export of NR4A1 which forms a proapoptotic NR4A1-blc-2 complex [23]. They also indicate that later stage, metastatic serous tumors showed significantly lower NR4A1 expression, a finding that might seem at first counterintuitive to some of our studies. It is however possible that exact function of NR4A is very context-dependent and complex, and this should be the focus of further studies. It is of interest that a recent study by Zhou et al. showed that NR4A1 plays a role in TGF $\beta$-induced breast cancer cell migration (ie pro-oncogenic) and knockdown of NR4A1 decreases basal and TGF $\beta$-induced migration in MDA-MB-231 cells [24]. Our findings here show that NR4A1 is present in both the cytoplasm and nucleus of several ovarian cells lines under normal growth conditions. Nuclear or cytoplasmic localization appears to vary depending on the specific HGSOC cell line analyzed. Previous studies have indicated that the phosphorylation state of NR4A1, which is regulated by the MAP/JNK kinase and Akt pathways, play a role in nuclear to cytoplasmic translocation of NR4A1 [23, 25-31]. The MAP, JNK, and Akt signaling pathways are highly dysregulated in cancer and it will be interesting to see if there is a correlation between alterations in these pathways and localization of NR4A1 in ovarian cell lines, which would be an important aspect to consider while attempting to clarify the relevant biology as well as in drug discovery.

Finally, the bi-functional activity of NR4A1 appears to be dependent on its sub-cellular location with nuclear localization being responsible for the mitogenic effects and mitochondrial localization being important for the pro-apoptotic effects [32]. Many pro-apoptotic agents and stimuli have been reported

307 to be associated with nuclear export and translocation of NR4A1 [17, 33]. Apoptosis induced in hepatocellular carcinoma cells by retinoids have been demonstrated to be NR4A1 dependent and to be 
associated with localization of NR4A1 in the mitochondria [34]. In androgen sensitive LNCaP

adenocarcinoma cells several apoptosis inducers consistently caused mitochondrial localization of

311 NR4A1, and mutants that lacked mitochondrial localization failed to induce apoptosis [33]. Cytosporone

312 B, a naturally occurring agonist of NR4A1, induces NR4A1 dependent apoptosis, modulates nuclear

313 export [35], and retards xenograft tumor growth by inducing NR4A1 translocation to the mitochondria in

314 gastric cancer cells [36]. It also demonstrates the bi-functional roles of NR4A1 in proliferation and

315 survival - its nuclear role as transcription factor with effects on gene expression, and its role in apoptosis

316 through its translocation to the mitochondria.

317 Developing a targeted molecule for the NR4A1 orphan nuclear receptor and applying the

318 compound in the clinic is very conceivable. Safe and colleagues were able to develop a molecule, 1,1-

319 bis(3-indolyl)-1-(p-substituted phenyl)methane (C-DIM) analog, which bind NR4A1 ligand binding

320 domain [37]. These compounds bind nuclear NR4A1 and inhibit several pro-oncogenic NR4A1-regulated

321 genes/pathways resulting in inhibition of mTOR signaling induction of oxidative and endoplasmic

322 reticulum stress and inhibition of several growth promoting and survival genes [19, 38-40]. C-DIMs are

323 synthetic analogs derived from indole-3-carbinol, which is a chemo-protective photochemical present in

324 leafy green vegetables. Future studies should test whether C-DIM/NR4A1 antagonists and other NR4A1-

325 targeting agents are effective for HGSOC cells, with the ultimate goal of targeting NR4A1 in a subset of

326 ovarian cancer patients.

327

328

\section{Acknowledgements}

The authors would like to acknowledge Archana Ramgopal; Jian Chen; Mildred Duvet; Rebecca Watters, PhD; Ryan Hartmaier, PhD; Dani Hochbaum, PhD; Carlos Castro, MD; John Comerci, MD; and the MWRI Core for their support and skillful technical assistance of this project. The study was supported by DOD CDMRP W81XWH-13-1-0205 (PI: SO), and by shared facility "Cancer Bioinformatics Service" that is supported in part by award P30CA047904. 


\section{REFERENCES}

[1] Siegel R, Naishadham D, Jemal A. Cancer statistics, 2013. CA: a cancer journal for clinicians. 2013;63:11-30.

[2] Makar AP. Hormone therapy in epithelial ovarian cancer. Endocrine-related cancer. 2000;7:85-93.

[3] Barakat RR. Principles and Practice of Gynecologic Oncology: LWW; 2009.

[4] Burger RA, Brady MF, Bookman MA, Fleming GF, Monk BJ, Huang H, et al. Incorporation of bevacizumab in the primary treatment of ovarian cancer. The New England journal of medicine. 2011;365:2473-83.

[5] Osborne CK, Zhao H, Fuqua SA. Selective estrogen receptor modulators: structure, function, and clinical use. Journal of clinical oncology : official journal of the American Society of Clinical Oncology. 2000;18:3172-86.

[6] Chen T. Nuclear receptor drug discovery. Current opinion in chemical biology. 2008;12:418-26.

[7] Sonoda J, Pei L, Evans RM. Nuclear receptors: decoding metabolic disease. FEBS letters. 2008;582:29.

[8] Gelman L, Feige JN, Desvergne B. Molecular basis of selective PPARgamma modulation for the treatment of Type 2 diabetes. Biochimica et biophysica acta. 2007;1771:1094-107.

[9] Yager JD, Davidson NE. Estrogen carcinogenesis in breast cancer. The New England journal of medicine. 2006;354:270-82.

[10] Augello MA, Den RB, Knudsen KE. AR function in promoting metastatic prostate cancer. Cancer metastasis reviews. 2014.

[11] Shao W, Brown M. Advances in estrogen receptor biology: prospects for improvements in targeted breast cancer therapy. Breast cancer research : BCR. 2004;6:39-52.

[12] Tseng GC, Wong WH. Tight clustering: a resampling-based approach for identifying stable and tight patterns in data. Biometrics. 2005;61:10-6.

[13] Tsai YS, Lin CT, Tseng GC, Chung IF, Pal NR. Discovery of dominant and dormant genes from expression data using a novel generalization of SNR for multi-class problems. BMC bioinformatics. 2008;9:425.

[14] Weroha SJ, Becker MA, Enderica-Gonzalez S, Harrington SC, Oberg AL, Maurer MJ, et al. Tumorgrafts as in vivo surrogates for women with ovarian cancer. Clin Cancer Res. 2014;20:1288-97. [15] Livak KJ, Schmittgen TD. Analysis of relative gene expression data using real-time quantitative PCR and the 2(-Delta Delta C(T)) Method. Methods. 2001;25:402-8.

[16] Maglott D, Ostell J, Pruitt KD, Tatusova T. Entrez Gene: gene-centered information at NCBI. Nucleic Acids Res. 2007;35:D26-31.

[17] Safe S KK, Li X and Lee S. NR4A orphan receptors and cancer. Nucl Recept Signal. 2011;9.

[18] Cho SD, Yoon K, Chintharlapalli S, Abdelrahim M, Lei P, Hamilton S, et al. Nur77 agonists induce proapoptotic genes and responses in colon cancer cells through nuclear receptor-dependent and nuclear receptor-independent pathways. Cancer Res. 2007;67:674-83.

[19] Lee SO, Abdelrahim M, Yoon K, Chintharlapalli S, Papineni S, Kim K, et al. Inactivation of the orphan nuclear receptor TR3/Nur77 inhibits pancreatic cancer cell and tumor growth. Cancer Res. 2010;70:6824-36.

[20] Wu H, Lin Y, Li W, Sun Z, Gao W, Zhang H, et al. Regulation of Nur77 expression by beta-catenin and its mitogenic effect in colon cancer cells. FASEB J. 2011;25:192-205.

[21] Inamoto T, Czerniak BA, Dinney CP, Kamat AM. Cytoplasmic mislocalization of the orphan nuclear receptor Nurr1 is a prognostic factor in bladder cancer. Cancer. 2010;116:340-6.

[22] Niu G, Lu L, Gan J, Zhang D, Liu J, Huang G. Dual roles of orphan nuclear receptor TR3/Nur77/NGFI-B in mediating cell survival and apoptosis. Int Rev Cell Mol Biol. 2014;313:219-58. 
[23] Wilson AJ, Liu AY, Roland J, Adebayo OB, Fletcher SA, Slaughter JC, et al. TR3 modulates platinum resistance in ovarian cancer. Cancer Res. 2013;73:4758-69.

[24] Zhou F, Drabsch Y, Dekker TJ, de Vinuesa AG, Li Y, Hawinkels L, et al. Nuclear receptor NR4A1 promotes breast cancer invasion and metastasis by activating TGF-beta signalling. Nature communications. 2014;5:3388.

[25] Fahrner TJ, Carroll SL, Milbrandt J. The NGFI-B protein, an inducible member of the thyroid/steroid receptor family, is rapidly modified posttranslationally. Molecular and cellular biology. 1990;10:6454-9. [26] Holmes WF, Soprano DR, Soprano KJ. Elucidation of molecular events mediating induction of apoptosis by synthetic retinoids using a CD437-resistant ovarian carcinoma cell line. The Journal of biological chemistry. 2002;277:45408-19.

[27] Pekarsky Y, Hallas C, Palamarchuk A, Koval A, Bullrich F, Hirata Y, et al. Akt phosphorylates and regulates the orphan nuclear receptor Nur77. Proceedings of the National Academy of Sciences of the United States of America. 2001;98:3690-4.

[28] Masuyama N, Oishi K, Mori Y, Ueno T, Takahama Y, Gotoh Y. Akt inhibits the orphan nuclear receptor Nur77 and T-cell apoptosis. The Journal of biological chemistry. 2001;276:32799-805.

[29] Holmes WF, Soprano DR, Soprano KJ. Early events in the induction of apoptosis in ovarian carcinoma cells by CD437: activation of the p38 MAP kinase signal pathway. Oncogene. 2003;22:637786.

[30] Han YH, Cao X, Lin B, Lin F, Kolluri SK, Stebbins J, et al. Regulation of Nur77 nuclear export by c-Jun N-terminal kinase and Akt. Oncogene. 2006;25:2974-86.

[31] Chen HZ, Zhao BX, Zhao WX, Li L, Zhang B, Wu Q. Akt phosphorylates the TR3 orphan receptor and blocks its targeting to the mitochondria. Carcinogenesis. 2008;29:2078-88.

[32] Kolluri SK, Bruey-Sedano N, Cao X, Lin B, Lin F, Han YH, et al. Mitogenic effect of orphan receptor TR3 and its regulation by MEKK1 in lung cancer cells. Molecular and cellular biology. 2003;23:8651-67. [33] Li H, Kolluri SK, Gu J, Dawson MI, Cao X, Hobbs PD, et al. Cytochrome c release and apoptosis induced by mitochondrial targeting of nuclear orphan receptor TR3. Science. 2000;289:1159-64. [34] Yang H, Nie Y, Li Y, Wan YJ. ERK1/2 deactivation enhances cytoplasmic Nur77 expression level and improves the apoptotic effect of fenretinide in human liver cancer cells. Biochemical pharmacology. 2011;81:910-6.

[35] Lee SO, Li X, Khan S, Safe S. Targeting NR4A1 (TR3) in cancer cells and tumors. Expert opinion on therapeutic targets. 2011;15:195-206.

[36] Zhan Y, Du X, Chen H, Liu J, Zhao B, Huang D, et al. Cytosporone B is an agonist for nuclear orphan receptor Nur77. Nature chemical biology. 2008;4:548-56.

[37] Lee SO, Li X, Hedrick E, Jin UH, Tjalkens RB, Backos DS, et al. Diindolylmethane Analogs Bind NR4A1 and Are NR4A1 Antagonists in Colon Cancer Cells. Molecular endocrinology (Baltimore, Md). 2014:me20141102. [38] Lee SO, Jin UH, Kang JH, Kim SB, Guthrie AS, Sreevalsan S, et al. The orphan nuclear receptor NR4A1 (Nur77) regulates oxidative and endoplasmic reticulum stress in pancreatic cancer cells. Mol Cancer Res. 2014;12:527-38.

[39] Hedrick E, Lee SO, Doddapaneni R, Singh M, Safe S. Nuclear receptor $4 A 1$ as a drug target for breast cancer chemotherapy. Endocr Relat Cancer. 2015;22:831-40.

[40] Hedrick E, Lee SO, Kim G, Abdelrahim M, Jin UH, Safe S, et al. Nuclear Receptor 4A1 (NR4A1) as a Drug Target for Renal Cell Adenocarcinoma. PLoS One. 2015;10:e0128308. 
427 Figure 1. TCGA in silico analysis. Figure 1A shows the heat map of nuclear receptor expression for tumors in the TCGA. Columns within the heat map represent TCGA tumor samples and rows represent individual nuclear receptors. The tight clustering algorithm utilized grouped the tumors into 5 clusters (see methods). Figure 1B shows progression-free and overall survival for the subset of patients with NR4A overexpression (cluster 1) as compared to those patients with lower NR4A expression. Median progression free survival of cluster 1 patients is significantly worse than that for those patients in clusters 2-5 $(p=0.03)$. While there was a trend towards worse overall survival for those patients in cluster 1 , the results did not reach statistical significance $(p=0.09)$.

Figure 2. mRNA NR4A1 expression in normal ovarian tissue and fallopian tube tissue. Figure 2A graphed as relative expression to $\beta$ actin. The expression of NR4A1 in the ovary is higher than most other tissues with the exception of skeletal muscle and tracheal tissue. Figure 2B demonstrates the immunohistochemical expression of NR4A1 in normal human ovarian tissue and fallopian tube tissue at 10x magnification. 1 demonstrates ovarian surface epithelium with underlying stroma while 2 demonstrates fallopian tube epithelium. The staining demonstrates strong nuclear NR4A1, but absence of 442 cytoplasmic NR4A1 in normal human ovarian and fallopian tube samples. Figure 3. NR4A1 immunohistochemical staining in benign and malignant ovarian tissue. Figure

$444 \quad 3 A$ is a table outlining histology of patient ovarian cancer samples analyzed and localization of NR4A1 staining. Positive NR4A1 staining was determined by observing at least $10 \%$ of cells in a given field with

446 brown staining in the nucleus or cytoplasm respectively. Figure 3B shows representative images of 447 immunohistochemical H\&E and NR4A1 staining. Representative images are shown with 20x 448 magnification and 40x insets. 
Figure 4. NR4A1 in human serous ovarian cancer cell lines. Figure 4A. Immunoblot for NR4A1 in whole cell lysate of several cell lines representing HGS OVCA. Figure 4B. Densitometry analysis on immunoblot in A showing OVCAR-432 and OVCAR3 have significantly higher NR4A1 expression.

452 Figure 4C. Representative immunofluorescent images of indicated cell lines stained for NR4A1 and 453 Hoechst. Images are representative of 20x magnification with a digital zoom inset. Figure 4D. Analysis 454 of total NR4A1 immunofluorescence signal in representative cell lines. Image analyses are representative of: PEO1(5 experiments), OVCA432(2 experiments), OVCAR8(3 experiments), OVSAHO(2 experiments), CA-OV-3(2 experiments), and OVCAR3(2 experiments). NR4A1 data from D was analyzed for nuclear/cytoplasmic localization. The fraction of NR4A1 in the cytoplasm was calculated by normalizing the integrated fluorescence intensity in the cytoplasm to the total integrated intensity in the cell (fluorescence in the cytoplasm and nucleus).

Figure 5. Quantitative Real-Time PCR data for total mRNA expression of NR4A1 in human patient derived xenografts of ovarian cancer. Figure 5A. Clinicopathological characteristics of the primary tumor sources used for generation of PDX models. Figure 5B. Gene expression corrected to respective

463 beta-actin expression, followed by normalization to NR4A1 delta-Ct of MCF7 in order to observe fold change compared to MCF7.

Table 1. Dominant and dormant driver NR genes from TCGA dataset. Gene expression of those NRs included in the TCGA analysis on high-grade serous ovarian cancers was analyzed using a "tight 467 clustering" algorithm (see methods section) and identified five patient clusters based on differential gene 468 expression. Driver genes of each cluster were investigated with the gene-dominant and gene-dormant 469 index method (21). This method extends the signal to noise ratio to identify features as either dominant 470 or dormant in a specific cluster compared to the other clusters. The associated table identifies those genes 471 included in the analysis and whether the gene of interest is considered dominant or dormant for each of 472 the 5 clusters. Statistically significant drivers are highlighted. 
Figure 1. TCGA in silico analysis.

476

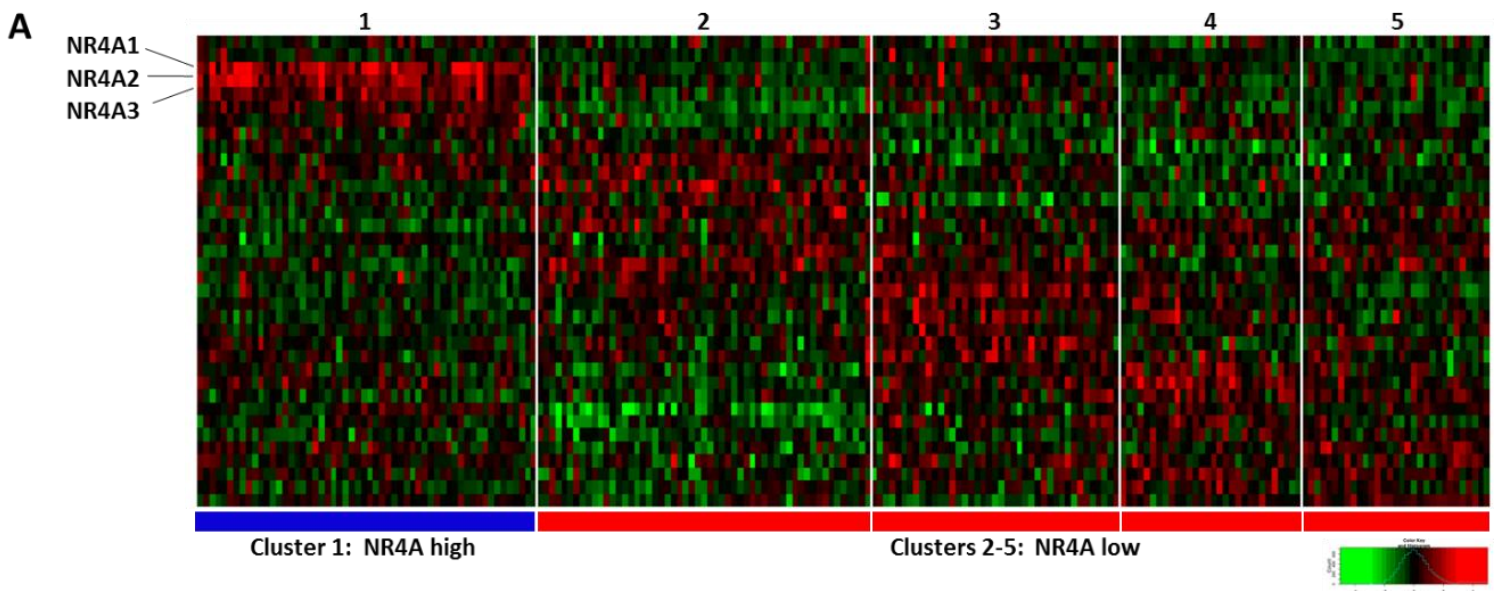

477

478

B

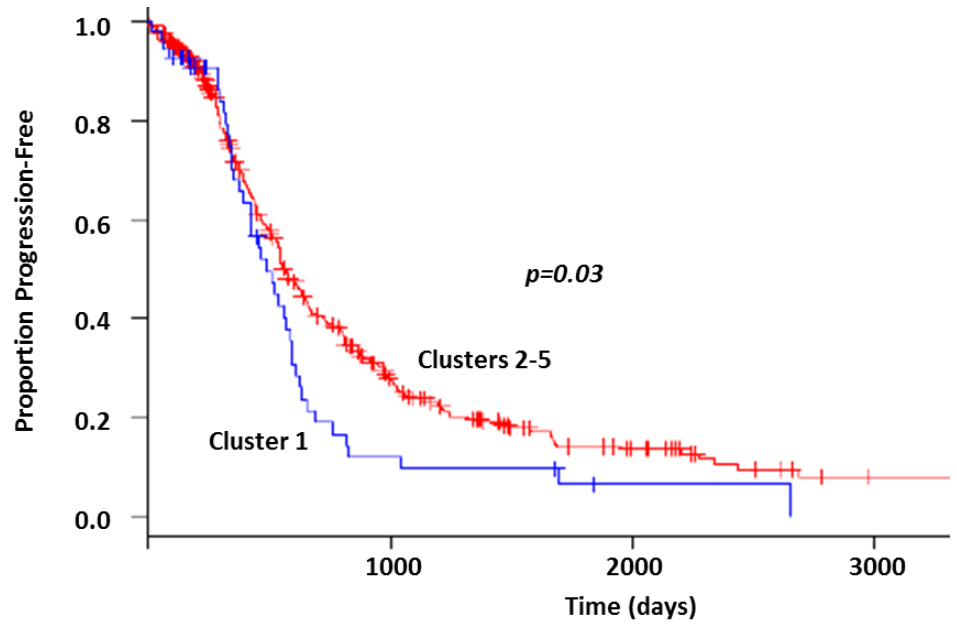

479 


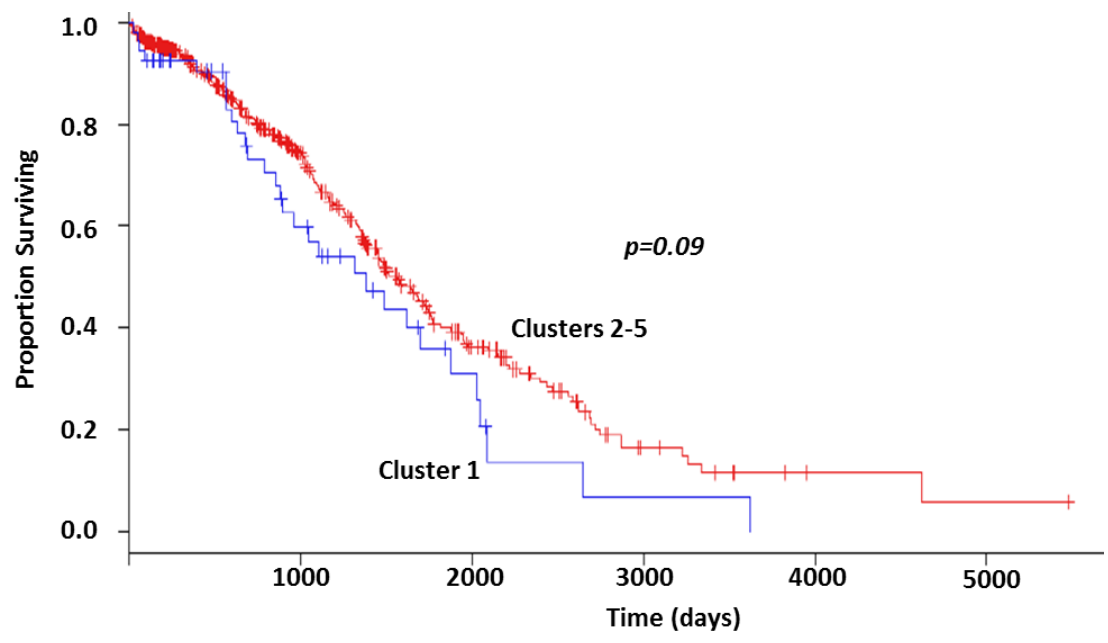

481

482

483 
Figure 2. mRNA NR4A1 expression in normal ovarian and fallopian tube tissue.

A

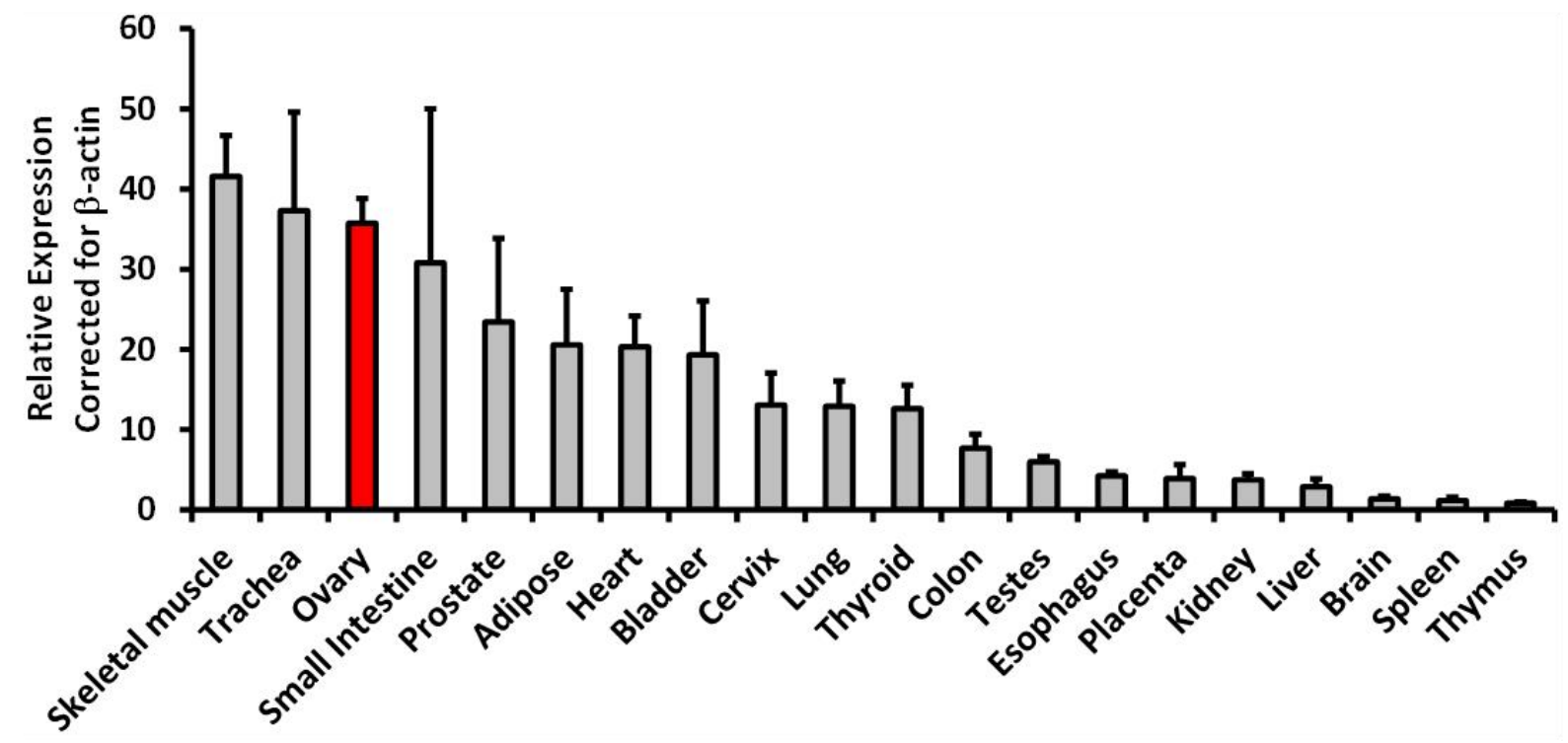

B

Normal Human Ovary

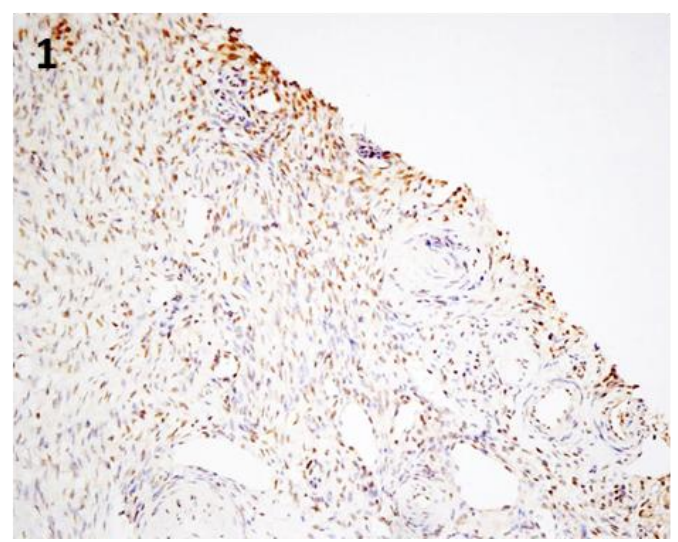

Normal Human Fallopian Tube

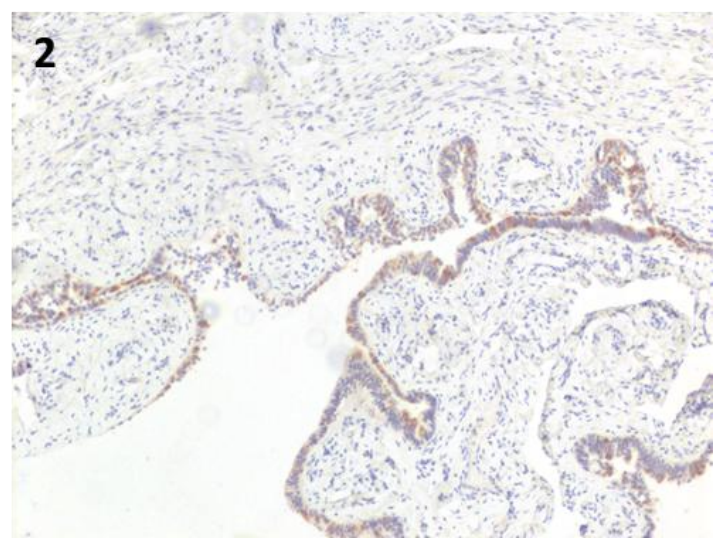


Figure 3. NR4A1 immunohistochemical staining in benign and malignant ovarian tissue.

\begin{tabular}{|c|c|c|c|c|c|}
\hline A & \multicolumn{2}{c}{ Total } & \multicolumn{2}{c}{$\begin{array}{c}\text { Nuclear } \\
\text { Only }\end{array}$} & \multicolumn{2}{c|}{$\begin{array}{c}\text { Cytoplasmic } \\
\text { Only }\end{array}$} & \multicolumn{2}{c|}{$\begin{array}{c}\text { Nuclear and } \\
\text { Cytoplasmic }\end{array}$} & None \\
\hline Benign & 10 & 10 & 0 & 0 & 0 \\
\hline Mucinous & 9 & 0 & 0 & 7 & 2 \\
\hline Endometrioid & 4 & 0 & 1 & 1 & 2 \\
\hline High Grade Serous & 6 & 0 & 0 & 5 & 1 \\
\hline
\end{tabular}
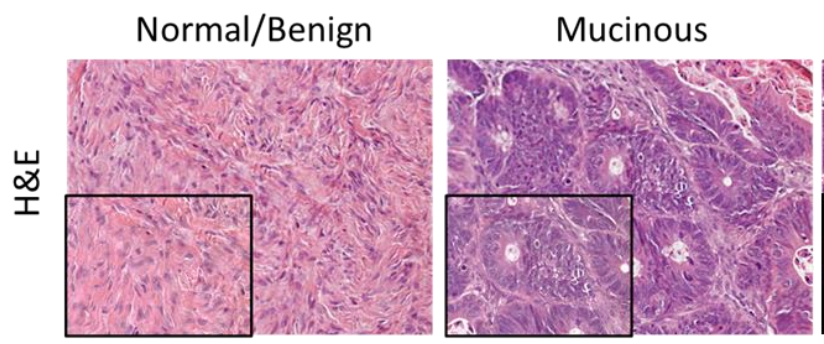

Endometrioid

High Grade Serous

\section{B}
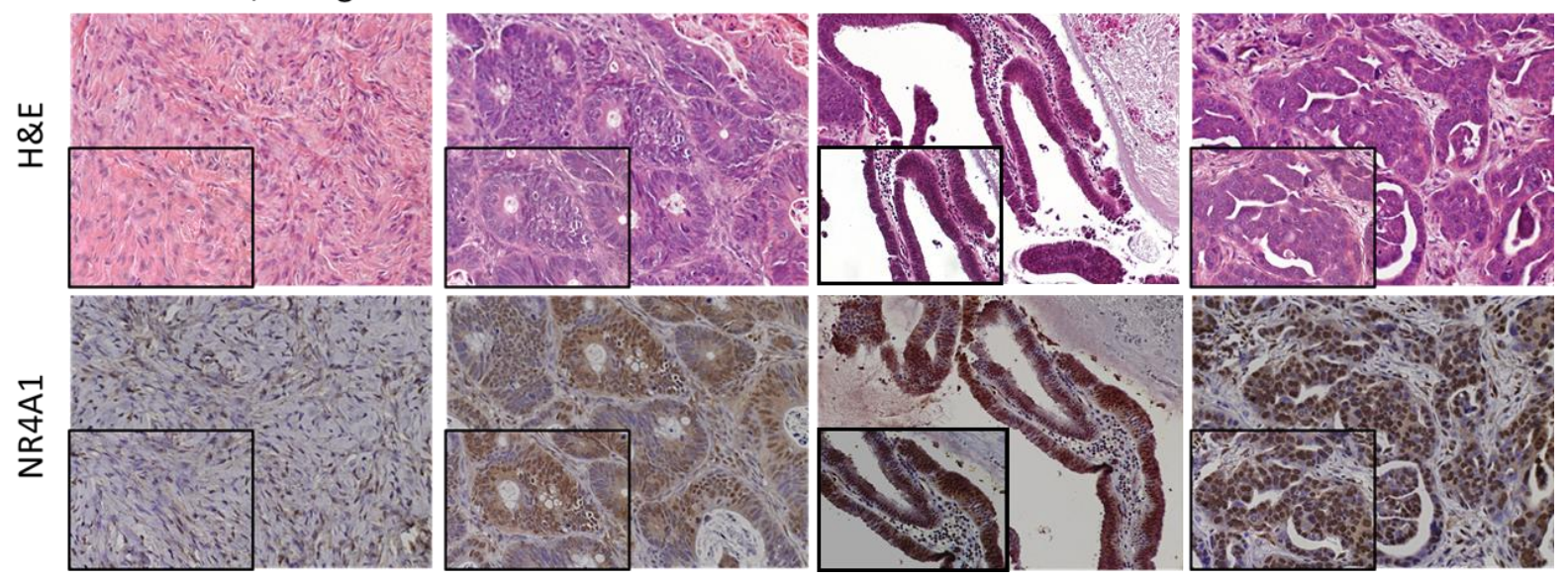

495 
Figure 4. NR4A1 in human serous ovarian cancer cell lines.
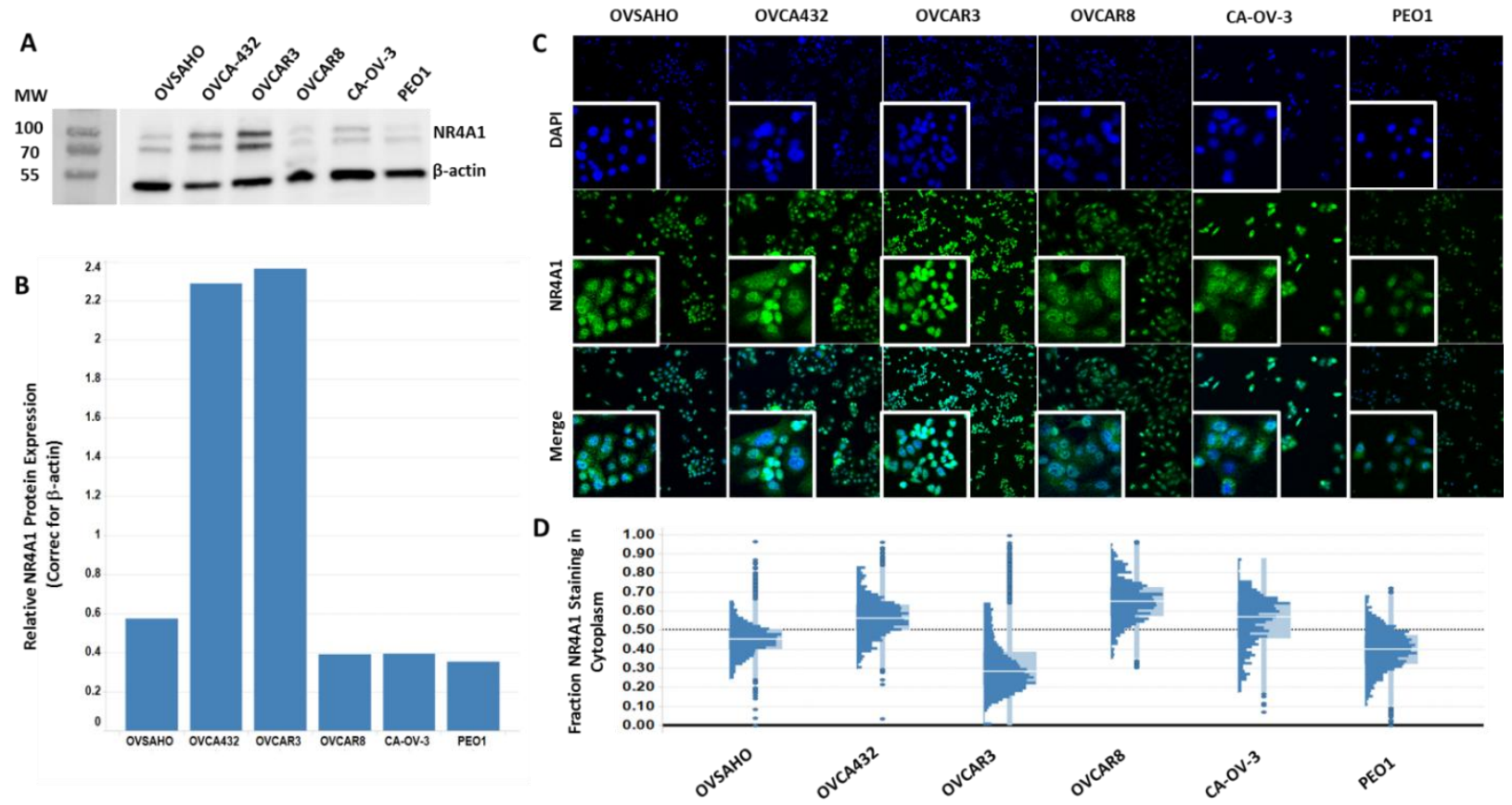
Figure 5. Quantitative Real-Time PCR data for total mRNA expression of NR4A1 in human patient derived xenografts of ovarian cancer.

A

\begin{tabular}{|c|c|c|c|}
\hline PH \# & Histology & $\begin{array}{c}\text { Median Age at } \\
\text { Diagnosis (y) }\end{array}$ & Final Stage \\
\hline $\begin{array}{c}13,26-27,32-33 \\
38,39,41-42,44- \\
45,48,51,54-55 \\
63,70,77\end{array}$ & Serous & 64 & $\begin{aligned} \mathrm{IC} & =1(6.25 \%) \\
\mathrm{II} & =1(6.25 \%) \\
\mathrm{IIC} & =8(50 \%) \\
\mathrm{IV} & =4(25 \%) \\
\mathrm{NR} & =2(12.5 \%)\end{aligned}$ \\
\hline 15 & Endometrioid & 79 & IIIC \\
\hline 3,6 & Carcinosarcoma & 68.5 & IIIC \\
\hline 18,29 & Mixed & 65.5 & IC \\
\hline 34 & Undifferentiated & 19 & IIIC \\
\hline
\end{tabular}

B

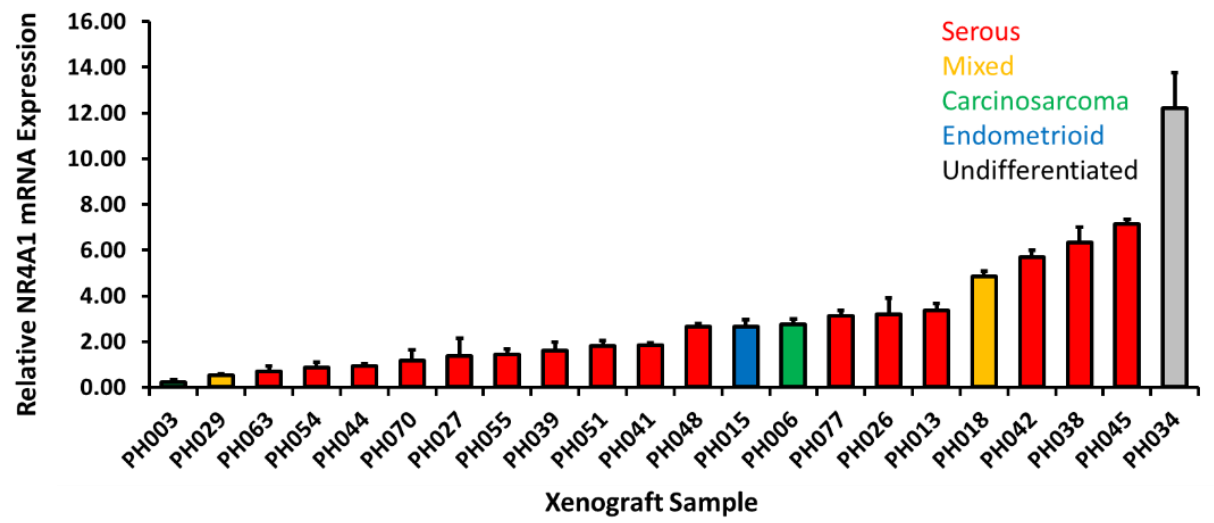


Table 1. Dominant and dormant driver NR genes from TCGA dataset.

\begin{tabular}{|c|c|c|c|c|}
\hline $\begin{array}{c}\text { Nuclear } \\
\text { Receptor }\end{array}$ & $\begin{array}{c}\text { Cluster } \\
\text { (Dominant) }\end{array}$ & p-value & $\begin{array}{c}\text { Cluster } \\
\text { (Dormant) }\end{array}$ & p-value \\
\hline AR & 5 & 0.1917 & 1 & 0.1431 \\
\hline ESR1 & 4 & 0.1938 & 1 & 0.7332 \\
\hline ESRRA & 3 & 0.5610 & 2 & 0.0001 \\
\hline HNF4A & 3 & $<0.0001$ & 5 & 0.1358 \\
\hline NR1D2 & 5 & $<0.0001$ & 3 & 0.0119 \\
\hline NR1H2 & 3 & 0.0220 & 1 & 0.1246 \\
\hline NR1H3 & 4 & 0.0488 & 2 & 0.7286 \\
\hline NR2C1 & 2 & 0.0004 & 3 & 0.0766 \\
\hline NR2C2 & 4 & 0.0801 & 1 & 0.5953 \\
\hline NR2E3 & 3 & 0.0189 & 5 & 0.0006 \\
\hline NR2F1 & 2 & 0.2902 & 4 & 0.0338 \\
\hline NR2F2 & 2 & 0.0021 & 4 & 0.4752 \\
\hline NR2F6 & 2 & 0.3093 & 4 & 0.6245 \\
\hline NR3C1 & 4 & 0.1591 & 2 & $<0.0001$ \\
\hline NR3C2 & 4 & $<0.0001$ & 2 & 0.0002 \\
\hline NR4A1 & 1 & $<0.0001$ & 4 & 0.1686 \\
\hline NR4A2 & 1 & $<0.0001$ & 2 & 0.2969 \\
\hline NR4A3 & 1 & $<0.0001$ & 5 & 0.5827 \\
\hline NR5A1 & 3 & 0.0077 & 5 & 0.0416 \\
\hline NR6A1 & 3 & 0.0128 & 1 & 0.1253 \\
\hline PGR & 2 & $<0.0001$ & 5 & 0.0495 \\
\hline PPARA & 4 & 0.0071 & 2 & 0.0003 \\
\hline PPARD & 3 & 0.0011 & 5 & 0.1183 \\
\hline RARA & 3 & $<0.0001$ & 4 & 0.2198 \\
\hline RARB & 1 & $<0.0001$ & 2 & 0.7096 \\
\hline RARG & 5 & 0.0002 & 2 & 0.0292 \\
\hline RORA & 1 & 0.8749 & 5 & 0.1937 \\
\hline RORC & 4 & 0.3249 & 1 & 0.2564 \\
\hline RXRA & 3 & 0.1722 & 2 & 0.0316 \\
\hline RXRB & 2 & 0.5655 & 1 & $<0.0001$ \\
\hline THRA & 2 & 0.7246 & 4 & 0.8499 \\
\hline THRB & 4 & 0.0181 & 2 & 0.0245 \\
\hline VDR & 1 & 0.0619 & 2 & 0.0076 \\
\hline GPER & 3 & 0.0012 & 1 & 0.8310 \\
\hline PGRMC1 & 2 & 0.0027 & 4 & 0.0119 \\
\hline PGRMC2 & 4 & 0.8194 & 3 & 0.0078 \\
\hline
\end{tabular}

506

507

508

509 
Table 1. Dominant and dormant driver NR genes from TCGA dataset.

\begin{tabular}{|c|c|c|c|c|}
\hline $\begin{array}{l}\text { Nuclear } \\
\text { Receptor }\end{array}$ & $\begin{array}{c}\text { Cluster } \\
\text { (Dominant) }\end{array}$ & p-value & $\begin{array}{c}\text { Cluster } \\
\text { (Dormant) }\end{array}$ & p-value \\
\hline$A R$ & 5 & 0.1917 & 1 & 0.1431 \\
\hline ESR1 & 4 & 0.1938 & 1 & 0.7332 \\
\hline ESRRA & 3 & 0.5610 & 2 & 0.0001 \\
\hline HNF4A & 3 & $<0.0001$ & 5 & 0.1358 \\
\hline NR1D2 & 5 & $<0.0001$ & 3 & 0.0119 \\
\hline NR1H2 & 3 & 0.0220 & 1 & 0.1246 \\
\hline NR1H3 & 4 & 0.0488 & 2 & 0.7286 \\
\hline NR2C1 & 2 & 0.0004 & 3 & 0.0766 \\
\hline NR2C2 & 4 & 0.0801 & 1 & 0.5953 \\
\hline NR2E3 & 3 & 0.0189 & 5 & 0.0006 \\
\hline NR2F1 & 2 & 0.2902 & 4 & 0.0338 \\
\hline NR2F2 & 2 & 0.0021 & 4 & 0.4752 \\
\hline NR2F6 & 2 & 0.3093 & 4 & 0.6245 \\
\hline NR3C1 & 4 & 0.1591 & 2 & $<0.0001$ \\
\hline NR3C2 & 4 & $<0.0001$ & 2 & 0.0002 \\
\hline NR4A1 & 1 & $<0.0001$ & 4 & 0.1686 \\
\hline NR4A2 & 1 & $<0.0001$ & 2 & 0.2969 \\
\hline NR4A3 & 1 & $<0.0001$ & 5 & 0.5827 \\
\hline NR5A1 & 3 & 0.0077 & 5 & 0.0416 \\
\hline NR6A1 & 3 & 0.0128 & 1 & 0.1253 \\
\hline PGR & 2 & $<0.0001$ & 5 & 0.0495 \\
\hline PPARA & 4 & 0.0071 & 2 & 0.0003 \\
\hline PPARD & 3 & 0.0011 & 5 & 0.1183 \\
\hline RARA & 3 & $<0.0001$ & 4 & 0.2198 \\
\hline RARB & 1 & $<0.0001$ & 2 & 0.7096 \\
\hline RARG & 5 & 0.0002 & 2 & 0.0292 \\
\hline RORA & 1 & 0.8749 & 5 & 0.1937 \\
\hline RORC & 4 & 0.3249 & 1 & 0.2564 \\
\hline RXRA & 3 & 0.1722 & 2 & 0.0316 \\
\hline RXRB & 2 & 0.5655 & 1 & $<0.0001$ \\
\hline THRA & 2 & 0.7246 & 4 & 0.8499 \\
\hline THRB & 4 & 0.0181 & 2 & 0.0245 \\
\hline VDR & 1 & 0.0619 & 2 & 0.0076 \\
\hline GPER & 3 & 0.0012 & 1 & 0.8310 \\
\hline PGRMC1 & 2 & 0.0027 & 4 & 0.0119 \\
\hline PGRMC2 & 4 & 0.8194 & 3 & 0.0078 \\
\hline
\end{tabular}

